\title{
Image manifold revealing for breast lesion segmentation in DCE-MRI
}

\author{
Liang $\mathrm{Hu}^{\mathrm{a}, \mathrm{b}}$, Zhaoning Cheng ${ }^{\mathrm{a}, \mathrm{b}}$, Manning Wang, ${ }^{\mathrm{a}, \mathrm{b},}$ and Zhijian Song ${ }^{\mathrm{a}, \mathrm{b}}$ \\ ${ }^{a}$ Digital Medical Research Center, Fudan University, Shanghai, China \\ ${ }^{\mathrm{b}}$ Shanghai Key Lab of Medical Image Computing and Computer Assisted Intervention, Shanghai, \\ China
}

\begin{abstract}
Dynamic contrast-enhanced magnetic resonance imaging (DCE-MRI) is widely used for breast lesion differentiation. Manual segmentation in DCE-MRI is difficult and open to viewer interpretation. In this paper, an automatic segmentation method based on image manifold revealing was introduced to overcome the problems of the currently used method. First, high dimensional datasets were constructed from a dynamic image series. Next, an embedded image manifold was revealed in the feature image by nonlinear dimensionality reduction technique. In the last stage, k-means clustering was performed to obtain final segmentation results. The proposed method was applied in actual clinical cases and compared with the gold standard. Statistical analysis showed that the proposed method achieved an acceptable accuracy, sensitivity, and specificity rates.
\end{abstract}

Keywords: Breast lesion, manifold learning, DCE-MRI, tumor segmentation, dimensionality reduction

\section{Introduction}

Dynamic contrast-enhanced magnetic resonance imaging (DCE-MRI) is a technology that enables the analysis of tumor blood vessels. After an initial scan is taken, a contrast agent is injected, and several dynamic scans are taken sequentially in regular intervals. By comparing the pre-contrast and postcontrast images, clinicians are able to identify cancerous areas and evaluate the extent of disease $[1,2]$.

Lesion segmentation plays an important role in breast-imaging interpretation. In a clinical setting, it is usually performed in subtracted images by manual delineation. Pre-contrast images are subtracted from post-contrast images to make lesion areas easier to recognize. However, it is labor-intensive and costly, and the segmentation results are subject to significant viewer interpretation.

Computer aided diagnosis (CAD) is helpful for breast-imaging translations [3-5]. Low level techniques include threshold and region growing [6]. The use of these methods is not very effective, and they are gradually becoming a preprocessing step completed before more advanced segmentation techniques are applied [7]. Automatic image segmentation relies on optimization procedures, and Markov random field (MRF) [8, 9], C-means cluster analysis [10], and level-set methods [11] are extremely data-dependent. These methods cannot guarantee that the segmentation results are anatomical-

\footnotetext{
* Address for correspondence: Manning Wang, Digital Medical Research Center, Fudan University, Shanghai, China. Tel.: +8621 54237181; Fax: +86 21 54237797; E-mail: mnwang@fudan.edu.cn.
}

0959-2989/15/\$35.00 @ 2015 - IOS Press and the authors. 
ly correct and can lead to misclassification. Alireza, [12] et al. compared different nonlinear dimensionality reduction methods in breast lesion segmentations and obtained highly accurate results. The technique involves images of different parameters, and the basis of the method is the segmentation result in the post-contrast image and itself is part of the input of the method, which seems not so reasonable.

To overcome the limitations of other methods, this paper proposed a new approach of manifold revealing to achieve better segmentation results with DCE-MRI data. Based on high dimensional dataset construction and nonlinear dimensionality reduction [13], the related idea is described in a previous study [14] on multi-modal image registration and it also proved to be effective for DCE-MRI segmentation.

\section{Methods}

\subsection{Data acquisition}

Clinical cases of 36 breast DCE-MRI were collected and analyzed as part of the research in this study. It was approved by the ethics committees of hospitals in December 2013. No informed consent was required because the clinical cases were analyzed anonymously. The images were obtained on a Siemens Magnetom Essenza 1.5T MR System and a Siemens Magnetom Verio 3T MR system from two different hospitals. Fast low angle shot (FLASH) MR sequences (TR $=4.67 \mathrm{~ms}, \mathrm{TE}=1.66 \mathrm{~ms}$, flip angle $=10^{\circ}$ ) were employed. After the pre-contrast image was obtained, gadopentate dimeglumine (Gd-DTPA) was injected as a contrast agent $(0.2 \mathrm{mmol} / \mathrm{kg})$ at the flow rate of $3 \mathrm{ml} / \mathrm{s}$, followed by a 20 cc saline flush. Five post-contrast images were obtained with an interval of $50 \mathrm{~s}$. The sizes of the image matrices varied from $384 \times 384$ to $768 \times 768$, depending on the clinical needs. All images were corrected for bias field after acquisition, and a registration step in the Siemens Syngo workstation was performed for patient motion correction after the acquisition of the 4D MR series.

All the experiments were performed with an Intel i5-3470 multi-core processor, and results were produced in Matlab R2013b.

\subsection{Overview of manifold learning}

Manifold learning can be explained as follows: Consider a high dimensional dataset $X$ denoted by a $n \times D$ matrix, where $n$ is the number of points in the dataset, and $D$ is the size of dimensionality; $X$ consists of $x_{i} \in R^{D}(i \in\{1,2, \ldots, n\})$. Assume there is redundant information in the original $D$ dimensional matrix, and in fact, $X$ lies on a $d$-dimensional manifold $M$ which is embedded in $R^{D}$ $(d<<D)$. With the approach of manifold learning and revealing, the original dataset $X$ will be transformed to the $d$-dimensional space $R^{d}$, preserving the geometry of the dataset as much as possible. The new dataset can be denoted by $y_{i} \in R^{D}(i \in\{1,2, \ldots, n\})$.

\subsection{High dimensional dataset}

For every pixel in the region of interest (ROI) in a DCE-MRI, a unique time intensity curve (TIC) is formed by collecting the intensity values from different time points. Pixels with similar TIC types are 
very likely to be the same type of tissue. To make the differentiation of lesion areas easier, a high dimensional dataset was built by collecting the TIC of every pixel in the ROI. For one DCE-MRI case, $T$ is defined as the total number of time points, including the pre-contrast scan, and $n$ is the total number of pixels in the selected ROI. TIC for each pixel can be thought of as a data point in a $T$ dimensional space, with each dimension corresponding to the intensity value of one time point. Therefore, time series of the ROI can be represented as a $n \times T$ matrix in which each TIC is denoted by $x_{i} \in R^{T}(i \in\{1,2, \ldots, n\})$.

Typical TIC types are shown in Figure 1. Although the shapes of TIC can vary across a wide range of patterns $[15,16]$, in most cases, the TIC of the lesion area will appear to be like one of the typical curves. These TICs are assumed to be within a non-linear $t$-dimensional manifold $M$ embedded in $R^{T}$. Here, $t<<T$. Afterward, the dataset is transformed to a low-dimensional representation.

\subsection{Manifold revealing and feature image production}

Various nonlinear dimensionality reduction methods have been proposed for revealing the essence of manifold. Among the methods, Laplacian Eigenmaps (LE) [13] is relatively computationally efficient and effective, and was adopted for multi-modal image registration in previous work [14].

The algorithm of LE can be described in the following three steps:

Step 1: Construct a neighborhood graph $G$ from the original dataset $X$ in which each data point $x_{i}$ is connected to its $k$ nearest neighbors.

Step 2: The weight of Gaussian kernel function is computed if two data points are connected, and it is assigned to the edge between them:

$$
w_{i j}=e^{-\frac{\left\|x_{i}-x_{j}\right\|^{2}}{2 \sigma^{2}}}
$$

Where $\sigma$ is the variance. Otherwise, $w_{i j}=0$.

Step 3: Compute low-dimensional representation by minimizing the cost function:

$$
\phi(Y)=\sum_{i j}\left\|y_{i}-y_{j}\right\|^{2} w_{i j}
$$

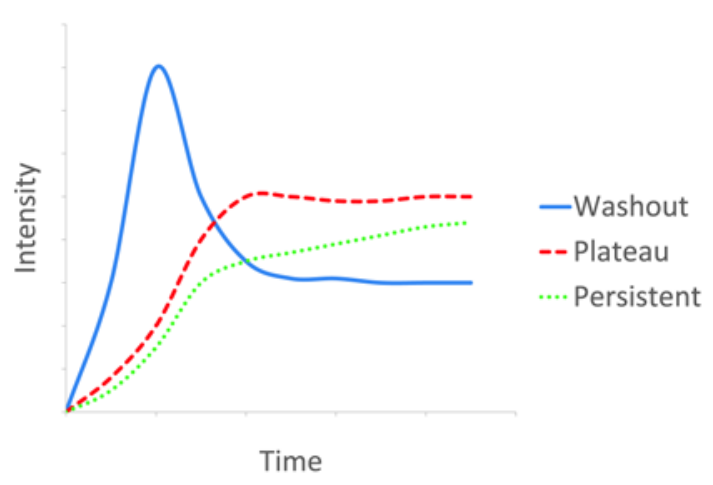

Fig. 1. Typical TIC types of DCE-MRI. 
The LE technique was performed on the high dimensional dataset constructed by pixel TIC and mapped from $T$-dimensional space to $t$-dimensional space. In this paper, $t$ was set to 3 . This means the $3 \mathrm{D}$ dataset was obtained and can be represented by RGB channels of the generated feature image while keeping the vascularity characteristics of the breast lesions unchanged.

\subsection{Segmentation and evaluation}

After manifold revealing, k-means clustering technique was performed on the generated 3D dataset for lesion differentiation. To evaluate the accuracy of this automatic method, the ground truth for segmentation was built first. In this study, a pre-step of auto threshold was carried out in subtracted images. After that, manual adjustments from three experienced specialists were performed on the results to obtain the gold standard.

Measurements of the accuracy rate, sensitivity, and specificity were given for quantitative evaluations. The intersection part of the proposed method and gold standard were denoted as True Positive (TP), the area of the proposed method outside the gold standard was False Positive (FP), the area of gold standard outside the proposed method was False Negative (FN), and the part outside both was True Negative (TN).

The accuracy rate (AR), sensitivity (SE), and specificity (SP) were defined as follows:

$$
\begin{gathered}
A R=\frac{T P+T N}{T P+T N+F P+F N} \times 100 \% \\
S E=\frac{T P}{T P+F N} \times 100 \% \\
S P=\frac{T N}{T N+F P} \times 100 \%
\end{gathered}
$$

\section{Experiment and results}

\subsection{Clinical case study}

The proposed method was applied on actual clinical studies for qualitative analyses. Figure 2 shows one case of an original breast DCE-MRI. The image marked ' 0 ' is the No. 19 slice of the pre-contrast volume from a patient, and images marked from ' 1 ' to ' 5 ' were taken from post-contrast scans. It can be seen that there is a lesion area in the breast with blurred boundaries. It is very difficult and timeconsuming for manual delineation of the lesion using the original time series.

Figure 3 shows the image processing results and the segmented lesion area. The subtracted image was produced by Image 2 and Image 0 in Figure 2. This is widely used in hospitals to help radiologists perform segmentation. The lesion area is more obvious in the subtracted image than the original series, but is still not clear enough for precise segmentation. To make segmentation less time-consuming, a feature image in RGB mode is produced to reveal the essence of the image manifold. The boundary of the lesion area is much clearer than in the subtracted image, and only the lesion area and the heart are highlighted in yellow, which reflects these two areas have similar contrast agent permeability. After a ROI is selected, k-means clustering technique is applied to the generated 3D dataset, and the breast lesion area is then segmented. The image of "Lesion Area" in Figure 3 shows the contours produced by the proposed method (in red) and the ground truth (in green). The two contours almost overlap. 


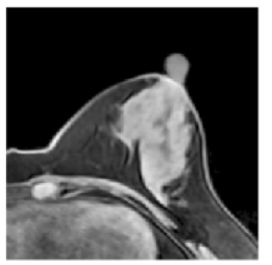

3

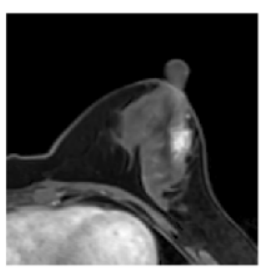

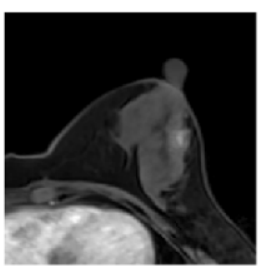

4

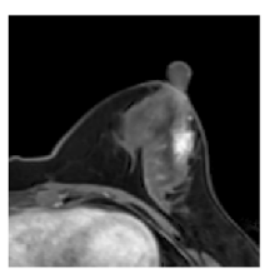

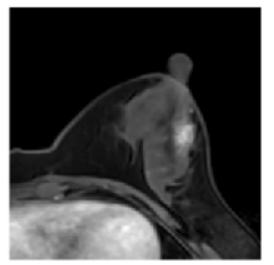

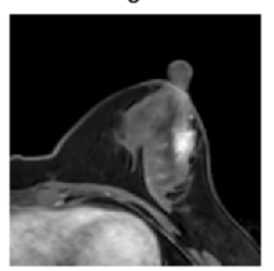

Fig. 2. Original DCE-MRI series.
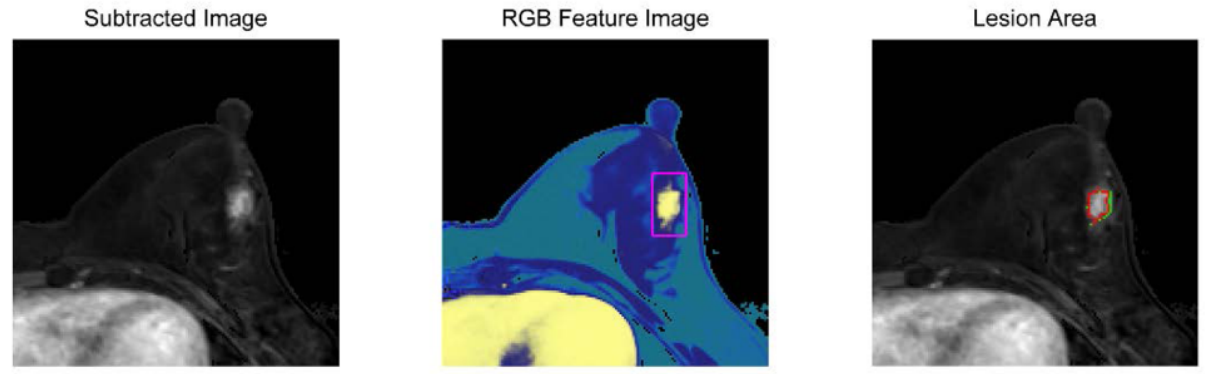

Fig. 3. Image processing results and segmented lesion area.

Scatter plots are shown in Figure 4. All of the pixels of the feature image were plotted as circles in the $3 \mathrm{D}$ coordinate system, with each axis representing one RGB channel. The RGB color value of the pixel was assigned to the corresponding circle in the scatter plot. It gave a visual guide to the locations of the lesion area and the heart. With the help of k-means clustering technique, the locations (green circles in the right image of Figure 4) were differentiated from other parts of the breast.

\subsection{Quantitative evaluation}

A two-step procedure was applied to each clinical case to build the gold standard for lesion segmentation:

Step 1: Threshold-based auto segmentation was performed on the subtracted images to generate the initial input.

Step 2: Three experienced radiologists consulted on the initial input to make manual adjustments to the segmented lesion boundary.

The segmentation results of the proposed approach were compared to the generated gold standard. The accuracy, sensitivity, and specificity rates were calculated according to Eqs. (3)-(5). The statistical 
results are listed in Table 1. For the 36 clinical cases, the proposed method yielded a high average accuracy rate of $0.97 \pm 0.01$. Also, it was found to have high specificity $(0.98 \pm 0.01)$ and acceptable sensitivity $(0.90 \pm 0.04)$. The computational time of the entire process varied from 3 to $12 \mathrm{~s}$, depending on the size of the image and the ROI.

\section{Discussion and conclusions}

Lesion areas in DCE-MRI usually have ambiguous boundaries, which are difficult to segment. Manual delineation is time-consuming and costly, and also allows for significant viewer variability. In this paper, an automatic manifold-based approach was proposed to solve these shortcomings. Pixel values from the time series of DCE-MRI were collected and constructed into a high-dimensional dataset. Nonlinear dimensionality reduction technique was employed to reveal the embedded image manifold. Then, 3D representation was obtained to build RGB channels of feature images, which were helpful for the differentiation of tissues with different permeability levels. After the completion of kmeans clustering, the lesion areas were segmented successfully.

Although vascularity and permeability varies across different types of lesions and individuals, they remain constant for the same tissue in the same patient. Lesion areas and normal tissues will have entirely different TIC in the same DCE-MRI series, which are differentiable in feature images. The proposed approach was validated in clinical cases and proved to be effective in reflecting the essential nature of the original dynamic image series. This method presented clear lesion boundaries in the generated feature images, as well as heart regions. This was due to the fact that lesion areas and hearts have similar tissue permeability and can be distinguished from other parts of the image.
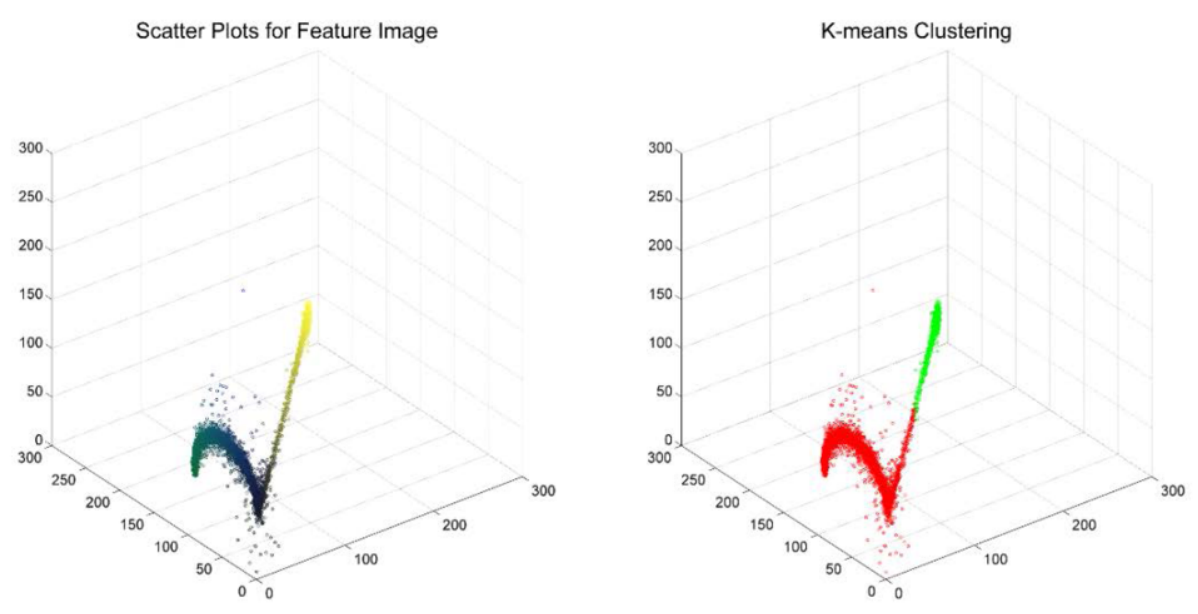

Fig. 4. Scatter plots and clustering technique.

Table 1

Mean value and standard deviation of AR, SE and SP*

\begin{tabular}{lll}
\hline AR (Mean \pm SD) & SE (Mean \pm SD) & SP (Mean \pm SD) \\
\hline $0.97 \pm 0.01$ & $0.90 \pm 0.04$ & $0.98 \pm 0.01$
\end{tabular}

Note: *36 clinical cases were collected; $\sigma=0.8$ in Gaussian kernel; AR: accuracy rate; SE: sensitivity; SP: specificity. 
The proposed method was also compared to a gold standard for quantitative evaluation and performed well statistically. Gold standard was established by the manual adjustments of three experienced radiologists on threshold-based segmentation results in subtracted images. Direct delineations in subtracted images were not performed because human interaction tends to produce smooth edges, which is often far from reality, and a pre-segmentation procedure also helps to lower labor costs.

Certainly, there are some limitations to this study. The algorithm has not been applied to 3D segmentation because the building of the ground truth for 3D segmentation is much more labor intensive, and requires more computational time using the proposed method. Improvements continue to be made on the performance of the algorithm, and will soon expand to 3D segmentation of full slices.

In summary, the proposed method introduces manifold learning in the construction of highdimensional datasets and reveals the essence of vascularity and permeability in breast DCE-MRI segmentation. It was applied on actual clinical cases and acquired acceptable accuracy, sensitivity, and specificity rates.

\section{Acknowledgments}

This study was partly supported by Project 81101128 and 81271670 , sponsored by the National Natural Science Foundation of China, National High Technology Research, and Development Program (No. 2012AA02A606), Key Technologies R\&D Program of China (No. 2012BAI14B05), Project 12441901600 supported by the Science and Technology Committee of Shanghai Municipality.

\section{References}

[1] M.D. Schnall, Breast MR imaging, Radiologic Clinics of North America 41 (2003), 43-50.

[2] E.A. Morris, Breast cancer imaging with MRI, Radiologic Clinics of North America 40 (2002), 443-466.

[3] D.J. Withey and Z.J. Koles, A review of medical image segmentation: Methods and available software, International Journal of Bioelectromagnetism 10 (2008), 125-148.

[4] K. Doi, Overview on research and development of computer-aided diagnostic schemes, Semin Ultrasound CT MR 25 (2004), 404-410.

[5] R.H. El Khouli, M.A. Jacobs and D.A. Bluemke, Magnetic resonance imaging of the breast, Seminars in Roentgenology 43 (2008), 265-281.

[6] S.Y. Wan and W.E. Higgins, Symmetric region growing, IEEE Trans Image Process 12 (2003), 1007-1015.

[7] J. Lai and Q. Wei. Automatic lung fields segmentation in CT scans using morphological operation and anatomical information, Bio-Medical Materials and Engineering 24 (2014), 335-340.

[8] R. Chellappa and A. Jain, Markov Random Fields Theory and Application, Academic Press, 1993.

[9] S. M'hiri, L. Cammoun and F. Ghorbel, Speeding up HMRF_EM algorithms for fast unsupervised image segmentation by bootstrap resampling: Application to the brain tissue segmentation, Signal Processing 87 (2007), 2544-2559.

[10] W. Chen, M.L. Giger and U. Bick, A fuzzy C-Means (FCM)-based approach for computerized segmentation of breast lesions in dynamic contrast-enhanced MR images, Academic Radiology 13 (2006), 63-72.

[11] X. Han, C. Xu and J.L. Prince, A topology preserving level set method for geometric deformable models, IEEE Transactions on Pattern Analysis and Machine Intelligence 25 (2003), 755-768.

[12] A. Akhbardeh and M.A. Jacobs, Comparative analysis of nonlinear dimensionality reduction techniques for breast MRI segmentation, Medical Physics 39 (2012), 2275-2289.

[13] M. Belkin and P. Niyogi, Laplacian eigen maps for dimensionality reduction and data representation, Neural Computation 15 (2003), 1373-1396.

[14] L. Hu, M.N. Wang and Z.J. Song, Manifold-based feature point matching for multi-modal image registration, International Journal of Medical Robotics and Computer Assisted Surgery 9 (2013), e10-e18.

[15] O. Kubassova, M. Boesen, R.D. Boyle, et al., Fast and robust analysis of dynamic contrast enhanced MRI datasets, Medical Image Computing and Computer-Assisted Intervention 10 (2007), 261-269. 
[16] C. Lavini, M.S. Buiter and M. Maas, Use of dynamic contrast enhanced time intensity curve shape analysis in MRI: Theory and practice, Reports in Medical Imaging 6 (2013), 71-82. 